\title{
Plasticidad fenotípica en dos poblaciones antárticas de Colobanthus quitensis (Caryophyllaceae) bajo un escenario simulado de cambio global
}

\section{Phenotypic plasticity in two antarctic populations of Colobanthus quitensis (Caryophyllaceae) under a simulated global change scenario}

\author{
Marco A. Molina-Montenegro ${ }^{1 *}$, Cristian Torres-Díaz ${ }^{2}$, Fernando Carrasco-Urra ${ }^{3}$, Leticia A. \\ GonZÁLEZ-SILVESTRE ${ }^{4}$ \& ERNESTO GIANOLI ${ }^{3,5,6}$
}

${ }^{1}$ Centro de Estudios Avanzados en Zonas Áridas (CEAZA), Facultad de Ciencias del Mar, Universidad Católica del Norte. Larrondo 1281, Coquimbo, Chile.

${ }^{2}$ Laboratorio de Genómica y Biodiversidad (LGB), Departamento de Ciencias Básicas, Facultad de Ciencias, Universidad del Bío-Bío, Chile.

${ }^{3}$ Departamento de Botánica, Facultad de Ciencias Naturales y Oceanográficas, Universidad de Concepción, Casilla 160-C, Chile.

${ }^{4}$ Centro de Estudios Avanzados en Zonas Áridas (CEAZA), Colina El Pino s/n, La Serena, Chile.

${ }^{5}$ Departamento de Biología, Universidad de La Serena, Casilla 599 La Serena, Chile.

${ }^{6}$ CASEB, Pontificia Universidad Católica de Chile, Alameda 340, Santiago, Chile.

marco.molina@ceaza.cl

\section{RESUMEN}

La Antártica se caracteriza por presentar condiciones ambientales extremas, lo cual limita el establecimiento de las plantas vasculares. Dentro de dichas características ambientales están las bajas temperaturas, corta estación de crecimiento y el estrés hídrico y nutricional. La baja disponibilidad hídrica ha sido sugerida como la principal limitante para el éxito de establecimiento, debido a que afecta de manera negativa las respuestas fisiológicas y reproductivas de las plantas. Colobanthus quitensis (Kunth) Bartl. (Caryophyllaceae) es una de las dos plantas vasculares nativas que habitan la Antártica Marítima y se ha demostrado que su desempeño fisiológico y reclutamiento es afectado negativamente por el estrés hídrico del suelo. La plasticidad fenotípica y la adaptación local serían dos de las principales estrategias de C. quitensis para lidiar con las adversas condiciones ambientales que predominan en el continente antártico. Se ha documentado que, bajo un escenario futuro de cambio climático, la disponibilidad hídrica en la Antártica aumentaría entre un 30-40\%, mejorando las condiciones para el establecimiento. En el presente trabajo evaluamos mediante un experimento de jardín común, realizado en cámaras de crecimiento, la plasticidad morfo-fisiológica y el esfuerzo reproductivo en individuos de $C$. quitensis provenientes de dos sitios en las Islas Shetland, bajo un escenario simulado de cambio climático (aumento en un $40 \%$ de la disponibilidad hídrica) y bajo la situación actual (disponibilidad hídrica registrada en los sitios de origen). En general, los individuos de C. quitensis de ambos sitios presentaron evidencia de plasticidad, aumentando su eficiencia fotoquímica, largo y ancho foliar, y esfuerzo reproductivo bajo la condición de aumento en la disponibilidad hídrica. La magnitud del aumento en la respuesta plástica fue mayor en aquellos individuos provenientes del sitio más árido en comparación a sus con-específicos del sitio más húmedo. Si los actuales patrones de cambio climático se mantienen, las condiciones abióticas podrían volverse más favorables para C. quitensis, mejorando su desempeño ecofisiológico y permitiendo la expansión de su rango de distribución sobre la Antártica.

Palabras clave: Antártica, esfuerzo reproductivo, estrés hídrico, Fv / Fm, islas Shetland.

\begin{abstract}
Antarctica is characterized by extreme environmental conditions, which limit the establishment of vascular plants. These environmental conditions include low temperatures, short growing season and shortage of water and nutrients. Low water availability has been suggested as the main constraint to successful establishment, because it adversely affects the reproductive and physiological responses of plants. Colobanthus quitensis (Kunth) Bartl. (Caryophyllaceae) is one of the two native vascular plants that inhabit the Maritime Antarctic and its physiological performance and recruitment have been shown to be negatively affected by soil water stress in the Antarctica. Phenotypic plasticity and local adaptation would
\end{abstract}


Plasticidad fenotípica en Colobanthus quitensis: Molina-Montenegro, M. ET AL.

be two of the main strategies of $C$. quitensis to cope with adverse environmental conditions prevailing in the Antarctic continent. It has been suggested that, under a future climate change scenario, water availability will increase by $30-40 \%$, improving the conditions for plant establishment. In this study we evaluated in a common garden experiment, conducted in growth chambers, the morpho-physiological plasticity and reproductive effort in C. quitensis individuals from two sites located in the Shetland Islands, under a simulated scenario of climate change ( $40 \%$ increase in water availability) and under the current situation (water availability recorded in the sites of origin). Overall, individuals of $C$. quitensis of both sites showed a plastic response, increasing the photochemical efficiency, leaf width and length and reproductive effort under increased water availability. Plasticity was greater in those individuals from the more arid site as compared to con-specifics from a mesic site. If current climate change patterns are maintained, abiotic conditions could become more favorable for $C$. quitensis, improving ecophysiological performance and allowing the spread of its range in the Antarctic.

KeYwords: Antarctic, water stress, reproductive effort, Fv / Fm, Shetland Islands.

\section{INTRODUCCIÓN}

El continente Antártico está entre los ambientes más estresantes para el desarrollo de la vida en la tierra (Convey et al. 2008). El establecimiento y supervivencia de plantas antárticas ocurre bajo severas condiciones, incluyendo bajas temperaturas, desecación por viento, alta radiación y una baja disponibilidad de nutrientes y agua en el suelo (Robinson et al. 2003, Wasley et al. 2006). Algunos estudios han demostrado que la baja disponibilidad hídrica sería el principal factor limitante para el éxito del establecimiento, germinación y desempeño fisiológico en las plantas antárticas (Convey 1996, Convey et al. 2003). Se ha documentado que el estrés hídrico induce cambios en atributos foliares (Grace 1997, Pedrol et al. 2000), disminución en el desempeño fisiológico (Flexas et al. 2006, Molina-Montenegro et al. 2011a) y reducción en el esfuerzo reproductivo (Rajakaruna et al. 2003, Molina-Montenegro et al. 2011b).

No obstante, las plantas poseen mecanismos para lidiar con las condiciones ambientales estresantes. La plasticidad fenotípica y la diferenciación ecotípica han sido sugeridos como los principales mecanismos que poseen las plantas para colonizar y establecerse en sitios con alto nivel de estrés (Sexton et al. 2002, Molina-Montenegro \& Cavieres 2010). La plasticidad fenotípica y la diferenciación ecotípica son dos estrategias complementarias, donde la plasticidad fenotípica puede inicialmente permitir el establecimiento de una especie, y una vez establecida la recombinación genética de fenotipos heredables podrían responder a la presión de selección local, obteniendo ecotipos adaptados con mayor adecuación biológica (Ellstrand \& Schierenbeck 2000, Sexton et al. 2002, Maron et al. 2004).

Se ha sugerido que la Antártica es uno de los ecosistemas más sensibles a los efectos del cambio climático global (IPCC 2007), debido a las condiciones climáticas que predominan en este continente. Algunas de las consecuencias del calentamiento global es el retroceso de los glaciales y el derretimiento del hielo en las capas superficiales del suelo (Turner et al. 2005), aumentando el área y agua disponible para las plantas, lo que favorecería la colonización. Para la Antártica se espera que, como consecuencia del cambio climático, no sólo aumente la temperatura de la superficie del suelo, sino también las precipitaciones anuales al estado líquido (Ye \& Mather 1997, IPCC 2007). Dependiendo del modelo a considerar, se prevé un incremento de $20-25 \%$ en las precipitaciones líquidas en el territorio antártico, siendo este efecto más intenso hacia el polo (IPCC 2007). Una de las principales características del territorio antártico -como se señalo previamente- es la escasez hídrica (Kennedy 1993, Beyer et al. 2000). Por ejemplo, Komárková et al. (1985) demostraron que la principal causa de mortalidad en especies vegetales presentes en el territorio antártico es la sequía estival. Así, un aumento en la disponibilidad hídrica podría mejorar las respuestas fisiológicas y reproductivas por parte de las plantas que habitan en esta zona.

No sólo el incremento en las precipitaciones líquidas inducidas por el aumento de la nubosidad aumentaría la disponibilidad de agua para las especies vegetales en la Antártica. Se ha documentado que el aumento de las temperaturas mejoraría la disponibilidad hídrica debido al descongelamiento del hielo presente en suelo y al aumento en la evaporación y posterior precipitación (Robinson et al. 2003). De esta manera, los efectos aditivos del aumento en las precipitaciones líquidas y descongelamiento del hielo podrían llegar a aumentar entre un 30-40\% la disponibilidad hídrica (Cullather et al. 1998, IPCC 2007), permitiendo un mejor desempeño fisiológico y un mayor éxito de establecimiento para las plantas que habitan estas zonas (Robinson et al. 2003).

La mayor parte del continente antártico permanece cubierto por nieve y hielo, dejando sólo un $2 \%$ de su superficie disponible para la colonización por plantas (Convey et al. 2008). Actualmente sólo dos plantas vasculares nativas están establecidas de manera natural en la Antártica Marítima, Deschampsia antarctica Desv. (Poaceae) y Colobanthus quitensis (Kunth) Bartl. (Caryophyllaceae) (Kappen 1999). Estudios previos realizados con estas especieshan demostrado que el estrés hídrico limita su rango de distribución y sus respuestas tanto reproductivas como fisiológicas (Leishman \& Wild 2001, Haussmann et al. 2009), siendo C. quitensis la especie con los límites espaciales más restringidos y con 
una mayor dependencia de la disponibilidad hídrica para su colonización y buen desempeño ecofisiológico (Fowbert \& Smith 1994, Convey 2003).

Dado que los modelos de cambio climático predicen incrementos en la disponibilidad hídrica en la Antártica, resulta relevante evaluar las potenciales respuestas de las plantas antárticas frente a las condiciones que se presume predominarán según las proyecciones climáticas. En el presente trabajo se evaluaron las respuestas fisiológicas, morfológicas y reproductivas de individuos de C. quitensis provenientes de dos sitios de la Antártica Marítima sometidos a una condición simulada de aumento en la disponibilidad hídrica. Específicamente nos hicimos las siguientes preguntas: i) ¿Aumenta el desempeño ecofisiológico en $C$. quitensis bajo un aumento en la disponibilidad hídrica del suelo?, y ii) ¿La respuesta ecofisiológica en C. quitensis será de mayor intensidad en aquellos individuos provenientes de un sitio más árido de la Antártica Marítima?

\section{MATERIALES Y MÉTODOS}

Sitios De ESTUdio

Los individuos experimentales de Colobanthus quitensis fueron recolectados en dos sitios con condiciones hídricas contrastantes. El primer sitio de estudio, denominado "Arctowski", se ubica en las inmediaciones de la Base Polaca "Henry Arctowski" en la Bahía Almirantazgo, isla Rey Jorge $\left(62^{\circ} 09^{\prime} \mathrm{S} ; 50^{\circ} 28^{\prime} \mathrm{W}\right)$. Este sitio se caracteriza por presentar una alta diversidad de musgos y líquenes además de una gran abundancia de las especies de plantas vasculares nativas Colobanthus quitensis y Deschampsia antarctica, así como algunos individuos de Poa annua (Olech \& Chwedorzewska 2011). El sitio de estudio corresponde a una franja paralela a la línea de costa con una suave pendiente dominada por un sustrato arenoso, con una altura promedio de $3 \mathrm{~m}$ s.n.m. con predominancia de fuertes vientos en dirección noroeste (Kejna 2008), lo que determinaría una alta demanda evaporativa para las especies vegetales y de un sustrato con baja disponibilidad hídrica. El segundo sitio de estudio, denominado "Hannah", se ubica en la costa sur del sector "Hannah Point" en la isla Livingston $\left(62^{\circ} 38^{\prime} \mathrm{S} ; 60^{\circ} 36^{\prime} \mathrm{O}\right)$. Este sitio se caracteriza por presentar marcadas pendientes en las laderas de los acantilados rocosos, cuyas alturas máximas alcanzan los 50-60 m s.n.m. Adicionalmente, este sitio se caracteriza por tener una gran cobertura de D. antarctica, y en menor abundancia de C. quitensis. Junto a estas especies es posible encontrar una alta abundancia de líquenes y musgos, probablemente debido a la alta disponibilidad hídrica del sustrato. Ambos sitios se ubican en las Islas Shetland del Sur. En esta zona la temperatura media anual es de $-2{ }^{\circ} \mathrm{C}$, con temperaturas que van desde -2 a $6{ }^{\circ} \mathrm{C}$ en verano y -1 a $-20{ }^{\circ} \mathrm{C}$ en invierno. La precipitación media anual bordea los
$700 \mathrm{~mm}$, principalmente en forma de nieve. Los suelos se caracterizan por estar formados por partículas minerales de gran tamaño (>1 mm), y bajos niveles de materia orgánica y acidez (Beyer et al. 2000).

\section{ESPECIE DE ESTUDIO}

Colobanthus quitensis (Kunth) Bartl. (Caryophyllaceae) es una de las dos plantas vasculares nativas establecidas que han colonizado exitosamente la Antártica Marítima y algunos sectores de la Península (Kappen 1999). Esta especie es autocompatible con reproducción sexual, no obstante la reproducción vegetativa es la principal forma de propagación de esta especie en la Antártica (Kennedy 1993). Se ha mostrado que esta especie posee una alta plasticidad en atributos morfológicos y fisiológicos, lo cual le ha permitido tener una amplia distribución, extendiéndose desde México $\left(17^{\circ} \mathrm{N}\right)$ hasta la Península Antártica (68 S) (Smith 2003).

CARACTERIZACIÓN HÍDRICA Y TAMAÑO DE LOS INDIVIDUOS EN LOS DIFERENTES SITIOS DE ESTUDIO

Para estimar las diferencias en la disponibilidad hídrica entre los dos sitios, 12 individuos de C. quitensis fueron seleccionados durante dos estaciones de crecimiento consecutivas, 2008-2009 y 2009-2010. El potencial hídrico del suelo se midió a una distancia de $10 \mathrm{~cm}$ de cada individuo mediante un tensiómetro digital (Jet Fill 2725 Series, Soil Moisture, Co, USA). Los tensiómetros se colocaron a 20 $\mathrm{cm}$ de profundidad y el potencial hídrico se registró después de $30 \mathrm{~min}$ de estabilización. Todas las mediciones fueron realizadas entre las 12:00-14:00 en días soleados.

Adicionalmente, durante la estación de crecimiento 2009-2010 se seleccionaron aleatoriamente 15 individuos adultos de $C$. quitensis de tamaño similar, los cuales fueron fotografiados para estimar su diámetro exacto $\left(\mathrm{cm}^{2}\right)$ como una estimación de éxito reproductivo. Para esto, a partir del primer individuo seleccionado se procedió a fotografiar el individuo más próximo que se encontrara al menos a $5 \mathrm{~m}$ de distancia del anterior. Para fotografiar cada individuo se utilizó un disco plástico de $10 \mathrm{~cm}$ de diámetro como escala para el posterior cálculo del tamaño. Finalmente, para obtener el área de los individuos, cada fotografía fue analizada con el programa analizador de imágenes "Sigma Scan pro 5.0".

\section{EXPERIMENTO SIMULADO DE CAMBIO GLOBAL}

Para evaluar las diferentes respuestas de individuos de $C$. quitensis provenientes de los dos sitios de estudio bajo un escenario futuro de cambio global, se recolectaron 30 individuos ( $7-10 \mathrm{~cm}$ de diámetro) en cada sitio y se trasladaron a cámaras de crecimiento ubicadas en el Centro de Estudios Avanzados en Zonas Áridas (CEAZA), La Serena, Chile. Los individuos fueron dispuestos en recipientes plásticos de 500 $\mathrm{ml}$ con una mezcla de turba: perlita: arena en una proporción de 1:1:1, por un periodo de aclimatación de 15 días con riego diario, fotoperiodo $16 / 8 \mathrm{~h}$ día/noche y a $10{ }^{\circ} \mathrm{C}$. Luego del 
periodo de aclimatación la mitad de los individuos de cada sitio fueron asignados de manera aleatoria a los siguientes tratamientos: 1) condición normal $\left(\mathrm{H}_{2} \mathrm{O}\right)$ la cual consistió en mantener la disponibilidad hídrica de cada sitio de origen y 2) cambio global $\left(+\mathrm{H}_{2} \mathrm{O}\right)$ la cual consistió en un aumento del $40 \%$ de la disponibilidad hídrica actual registrada en cada sitio. Para obtener este último valor se consideró un aumento del $40 \%$ en relación al promedio registrado en ambos sitios durante las dos estaciones de crecimiento antes señaladas. Para obtener la condición " $\mathrm{H}_{2} \mathrm{O}$ ", los individuos provenientes de Arctowski y de Hannah fueron irrigados cada 5 días con 30 y $50 \mathrm{ml}$, respectivamente. Para obtener la condición " $+\mathrm{H}_{2} \mathrm{O}$ ", los individuos de Arctowski y de Hannah fueron irrigados cada 5 días con 45 y $70 \mathrm{ml}$, respectivamente. Para controlar la disponibilidad hídrica en los recipientes plásticos se instalaron sensores de humedad (sondas ECHO EA-10, USA). Los individuos de C. quitensis de ambos sitios fueron mantenidos por un periodo de 70 días bajo las condiciones experimentales, terminado este periodo se les midió atributos fisiológicos, morfológicos y el porcentaje de floración.

\section{MEDICIONES MORFO-FISIOLÓGICAS Y ESFUERZO REPRODUCTIVO}

Luego de 70 días de exposición a los dos tratamientos hídricos, en 12 individuos de C. quitensis de cada sitio (n = 24 / sitio) se seleccionaron hojas totalmente desarrolladas y visualmente sanas para medir la eficiencia fotoquímica del fotosistema II (Fv / Fm) mediante un Fluorímetro portátil (Hansatech, Instruments Ltda. Norfolk, England). Cada hoja fue previamente oscurecida por un periodo de 45 min hasta obtener la totalidad de los centros de reacción abiertos. Consideramos la eficiencia máxima fotoquímica del fotosistema II $\left(\mathrm{Fv} / \mathrm{Fm}\right.$; donde $\mathrm{Fv}=\left[\mathrm{Fm}-\mathrm{F}_{0}\right]$, Fm $=$ fluorescencia máxima, y $\mathrm{F}_{0}=$ fluorescencia mínima) como una medida de desempeño fisiológico bajo una condición ambiental determinada (Maxwell \& Johnson 2000). Sobre los mismos individuos de C. quitensis utilizados para las mediciones de Fv / Fm, se seleccionaron 3 hojas centrales por individuo, a las cuales se les midió el largo $\mathrm{y}$ ancho con un caliper digital (Mitutoyo; resolución = $0.01 \mathrm{~mm}$ ). Posteriormente, los valores medidos en las 3 hojas fueron promediados, obteniéndose un valor por individuo. Finalmente se comparó el efecto del aumento de la disponibilidad hídrica sobre el esfuerzo reproductivo, medido como el porcentaje de floración. Para esto se registró, cada 3 días y por un período de 70 días, el número de flores producidas por cada individuo experimental.

\section{ANÁlisis ESTADÍSTICOS}

La humedad del suelo de ambos sitios durante las dos estaciones de crecimientos fue comparada mediante un análisis de varianza (ANOVA) de dos vías. El tamaño de los individuos de $C$. quitensis de ambos sitios fue comparado mediante un ANOVA de una vía. La eficiencia fotoquímica del fotosistema II (Fv / Fm), la longitud y ancho foliar fueron comparados mediante un ANOVA de dos vías, donde el sitio (Hannah vs. Arctowski) y el tratamiento hídrico $\left(\mathrm{H}_{2} \mathrm{O}\right.$ vs. $\left.+\mathrm{H}_{2} \mathrm{O}\right)$ fueron los factores. Para todos los ANOVA se evaluó la normalidad y homogeneidad de los datos mediante las pruebas de ShapiroWilks y Bartlett, respectivamente (Zar 1999).

\section{RESULTADOS}

CARACTERIZACIÓN HÍDRICA Y TAMAÑO DE LOS INDIVIDUOS EN LOS DIFERENTES SITIOS DE ESTUDIO

La disponibilidad hídrica del suelo fue significativamente mayor $\left(\mathrm{F}_{1,44}=476,97 ; \mathrm{p}<0,001\right)$ en Hannah que en Arctowski (Fig. 1). No se encontraron diferencias en la disponibilidad hídrica del suelo entre la estación de crecimiento 2008-2009 y 2009-2010 $\left(\mathrm{F}_{1,44}=7,56 ; \mathrm{p}=0,071\right)$. Tampoco hubo una interacción significativa de Sitio x Estación de crecimiento $\left(\mathrm{F}_{1,44}=0,1 ; \mathrm{p}<0,82\right.$; Fig. 1). Los individuos de C. quitensis provenientes de Hannah presentaron un tamaño promedio significativamente mayor $\left(\mathrm{F}_{1,28}=473,32 ; \mathrm{p}<0,001\right)$ que sus con-específicos de Arctowski, con promedios de 21,5 $\pm 2,2$ DE y 7,2 $\pm 1,3 \mathrm{DE}$, respectivamente.

MEDICIONES MORFO-FISIOLÓGICAS Y ESFUERZO REPRODUCTIVO La eficiencia fotoquímica del fotosistema II (Fv / Fm) fue significativamente mayor en individuos de Hannah $\left(\mathrm{F}_{1,28}=45,18 ; \mathrm{p}<0,001\right)$ y bajo una condición de mayor disponibilidad de agua $\left(\mathrm{F}_{1,28}=56,40 ; \mathrm{p}<0,001\right)$. El resultado

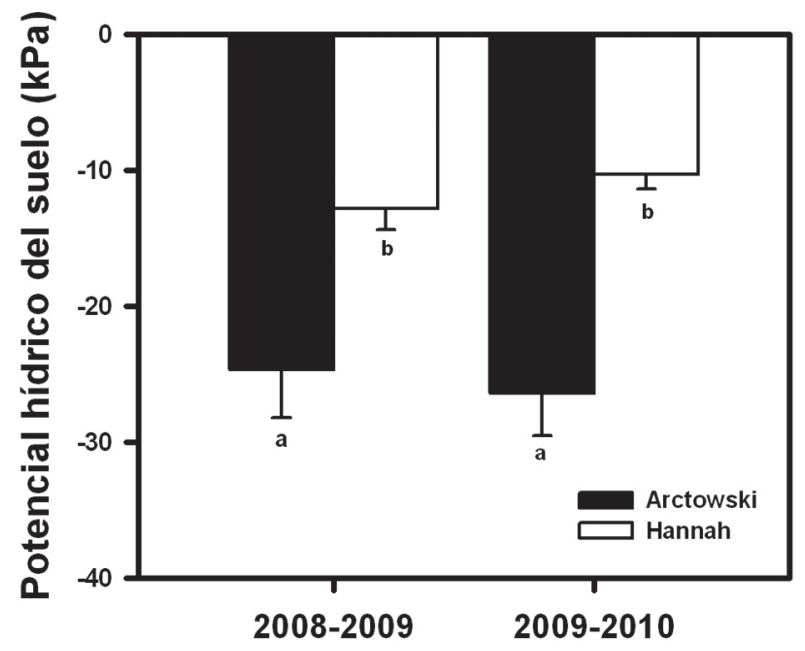

FIgURA 1. Potencial hídrico $(\mathrm{kPa})$ registrado en el suelo de Arctowski (barras negras) y Hannah (barras blancas) durante dos estaciones de crecimiento (2008-2009 y 2009-2010), islas Shetland del sur, Antártica. Las barras son promedios \pm 2 EE. (Letras diferentes indican diferencias significativas; prueba de Tukey, $\alpha=0.05$ ).

FIGURE 1. Soil matric potential $(\mathrm{kPa})$ recorded in the soil of Arctowski (filled bars) and Hannah (empty bars) during two growing seasons (2008-2009 and 2009-2010), South Shetland Island, Antarctic. Bars are means $\pm 2 \mathrm{EE}$. (Different letters indicate significant differences; Tukey test, $\alpha=0.05$ ). 
principal es que la interacción Sitio x Disponibilidad hídrica fue significativa $\left(\mathrm{F}_{1,28}=473,32 ; \mathrm{p}<0,001\right)$. Si bien la eficiencia fotoquímica aumentó con la disponibilidad de agua en los individuos de ambos sitios, este incremento fue de mayor magnitud en los individuos provenientes de Arctowski (Fig. 2).

La longitud foliar no fue diferente entre los individuos de C. quitensis provenientes de ambos sitios (Fig. 3A). Por otro lado, individuos en una condición de mayor disponibilidad hídrica presentaron hojas significativamente más largas $\left(\mathrm{F}_{1,36}\right.$ $=66,21 ; \mathrm{p}<0,001)$ que aquéllos bajo la condición control (Fig. 3A). La interacción Sitio x Disponibilidad hídrica fue significativa $\left(\mathrm{F}_{1,36}=6,99 ; \mathrm{p}=0,012\right)$, evidenciando que el incremento en el tamaño de las hojas de los individuos de Arctowski fue de mayor intensidad en respuesta a la variación en disponibilidad de agua (Fig. 3A). El ancho foliar no fue diferente entre los individuos provenientes de ambos sitios; no obstante, aquéllos expuestos a una mayor disponibilidad hídrica presentaron hojas con un ancho promedio mayor $\left(\mathrm{F}_{136}\right.$ $=0,68 ; \mathrm{p}=0,41$ y $_{1,36}=10,39 ; \mathrm{p}=0,002$, respectivamente). El factor de interacción Sitio $\mathrm{x}$ Disponibilidad hídrica fue significativo $\left(\mathrm{F}_{136}=66,21 ; \mathrm{p}<0,001\right)$, indicando que si bien los individuos de $C$. quitensis de ambos sitios aumentaron su ancho foliar bajo una condición de mayor disponibilidad hídrica, aquéllos provenientes de Arctowski lo hicieron en mayor grado (Fig. 3B).

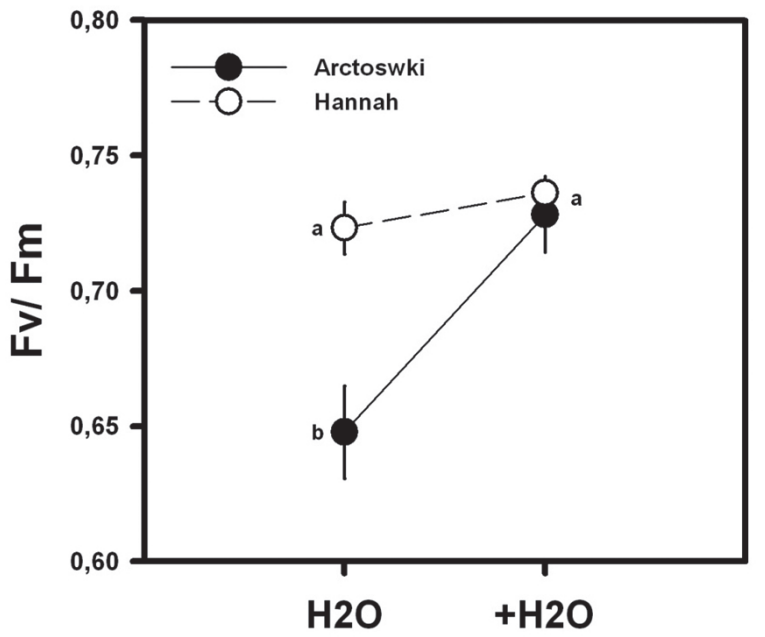

FIGURA 2. Eficiencia fotoquímica máxima del PSII (Fv / Fm) en individuos de Colobanthus quitensis provenientes de Arctowski (círculos negros) y Hannah (círculos blancos) expuestos a una condición hídrica normal $\left(\mathrm{H}_{2} \mathrm{O}\right)$ y bajo alta disponibilidad hídrica $\left(+\mathrm{H}_{2} \mathrm{O}\right)$. Los círculos representan los promedios \pm 2 EE. (Letras diferentes indican diferencias significativas; prueba de Tukey, $\alpha$ $=0.05$ ).

FIGURE 2. Maximal photochemical efficiency of PSII (Fv / Fm) in Colobanthus quitensis individuals from Arctowski (filled circles) and Hannah (empty circles) exposed to normal hydric condition $\left(\mathrm{H}_{2} \mathrm{O}\right)$ and high water availability $\left(+\mathrm{H}_{2} \mathrm{O}\right)$. Circles are means \pm 2 EE. (Different letters indicate significant differences; Tukey test, $\alpha=0.05)$.

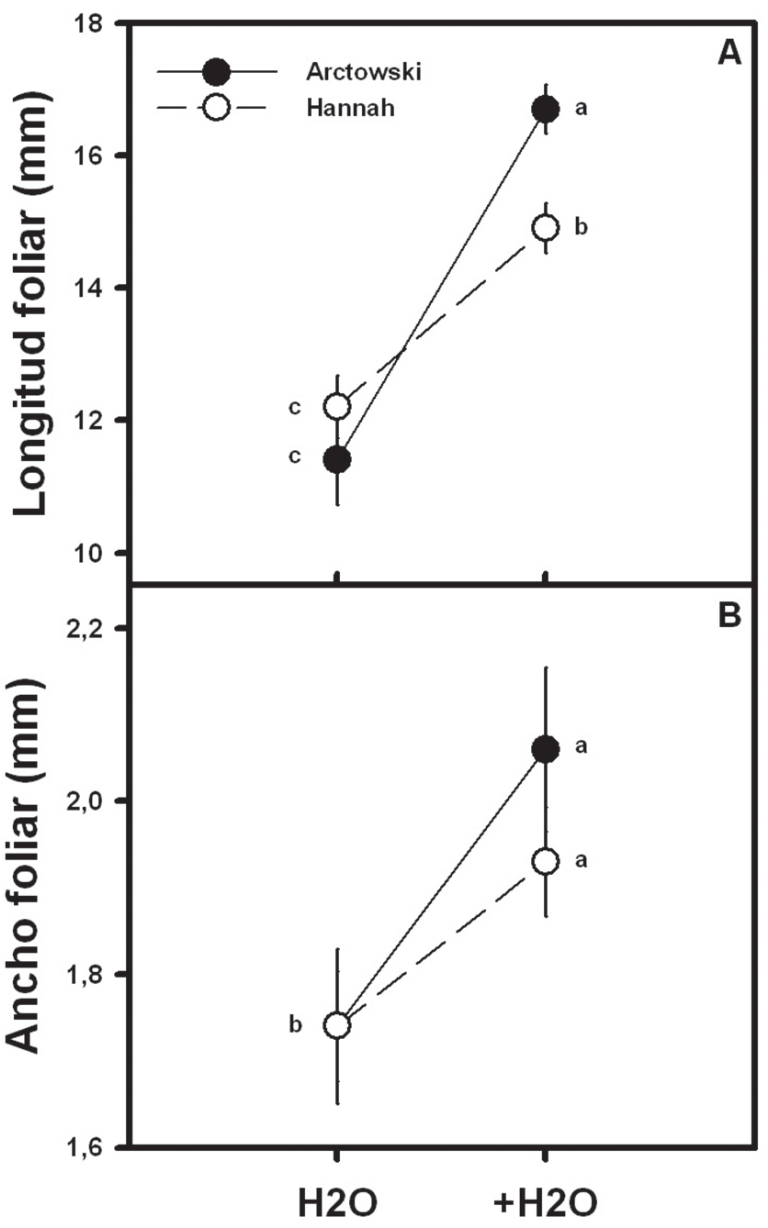

FIgURA 3. Longitud (A) y ancho foliar (B) en individuos de Colobanthus quitensis provenientes de Arctowski (círculos negros) y Hannah (círculos blancos) expuestos a una condición hídrica normal $\left(\mathrm{H}_{2} \mathrm{O}\right)$ y bajo alta disponibilidad hídrica $\left(+\mathrm{H}_{2} \mathrm{O}\right)$. Los círculos representan los promedios \pm 2 EE. (Letras diferentes indican diferencias significativas; test de Tukey, $\alpha=0.05$ ).

FIGURE 3. Length (A) and width of leaves (B) in Colobanthus quitensis individuals from Arctowski (filled circles) and Hannah (empty circles) exposed to normal hydric condition $\left(\mathrm{H}_{2} \mathrm{O}\right)$ and high water availability $\left(+\mathrm{H}_{2} \mathrm{O}\right)$. Circles are means $\pm 2 \mathrm{EE}$. (Different letters indicate significant differences; Tukey test, $\alpha=0.05$ ).

El porcentaje de floración de los individuos de $C$. quitensis provenientes de ambos sitios fue mayor bajo el tratamiento de mayor disponibilidad hídrica en comparación a sus con-específicos del tratamiento control (Fig. 4). El mayor porcentaje de floración fue alcanzado por los individuos de ambas poblaciones expuestos a una mayor disponibilidad hídrica, ambos con un 53\%, seguidos por los individuos de Hannah bajo la condición control, con un $40 \%$, y finalmente por los individuos provenientes de Arctowski bajo la condición control con un 27\% (Fig. 4). 


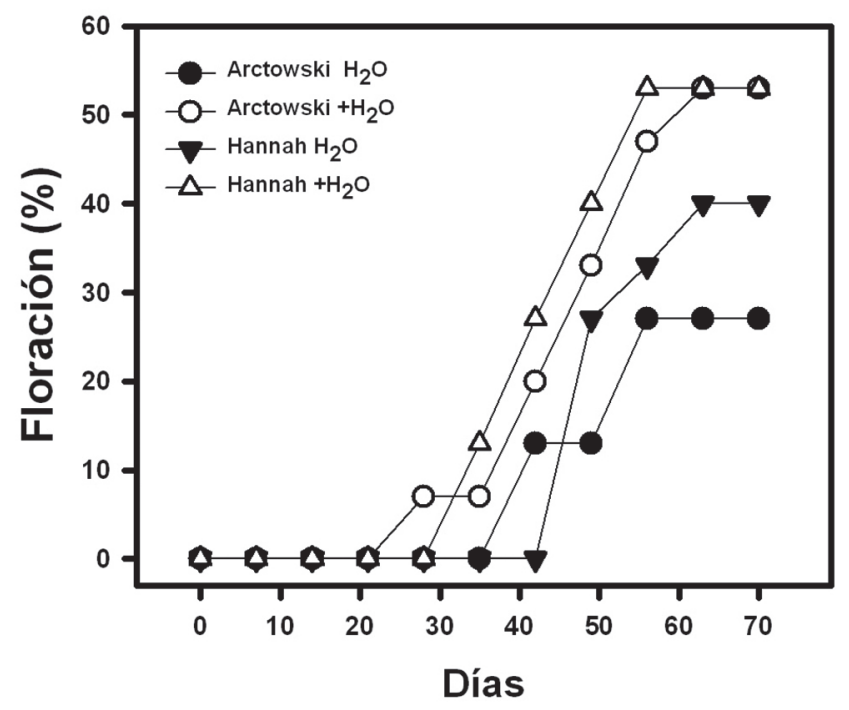

FIgURA 4. Variación del porcentaje de floración a través del tiempo en individuos de Colobanthus quitensis provenientes de Arctowski (círculos) y Hannah (triángulos) expuestos una la condición hídrica normal $\left(\mathrm{H}_{2} \mathrm{O}\right.$, símbolos negros) y bajo alta disponibilidad hídrica $\left(+\mathrm{H}_{2} \mathrm{O}\right.$, símbolos blancos).

FIGURE 4. Variation in flowering percentage along the time in Colobanthus quitensis individuals from Arctowski (circles) and Hannah (triangles) exposed to normal hydric condition $\left(\mathrm{H}_{2} \mathrm{O}\right.$, black symbols) and high water availability $\left(+\mathrm{H}_{2} \mathrm{O}\right.$, white symbols).

\section{DISCUSIÓN}

Las condiciones abióticas características del continente antártico determinan que el crecimiento, desempeño fisiológico y reproducción de las plantas que habitan esta zona se encuentren cerca de los límites para la vida (Robinson et al. 2003). Las principales limitaciones que las plantas deben sobrepasar para su establecimiento en este hábitat es la corta estación de crecimiento, bajas temperaturas, efecto desecante del viento, alta radiación UV y la baja disponibilidad de agua y nutrientes en el suelo (Robinson et al. 2003, Singh et al. 2009). Lo anterior, determina que la mayor parte de la vegetación antártica esté confinada a las áreas libres de hielo, las cuales son climáticamente más benignas, pero constituyen menos del $2 \%$ de la superficie del continente.

Se ha sugerido que uno de los atributos claves para una colonización exitosa en ambientes extremos es la plasticidad fenotípica, entendida como la capacidad de un individuo de generar un ajuste morfo-fisiológico que le permita establecerse y completar su ciclo de vida a lo largo de un rango de hábitats (Pigliucci 2001). Este podría ser el caso de Colobanthus quitensis, que como se muestra en este estudio, es capaz de modificar tanto su morfo-fisiología como su esfuerzo reproductivo bajo diferentes condiciones hídricas. Esta plasticidad le permitiría a $C$. quitensis responder rápidamente a la aparición de condiciones más favorables (e.g., aumento de la disponibilidad hídrica), lo cual provocaría un aumento en el desempeño fisiológico, crecimiento de estructuras vegetativas y un probable éxito en la colonización de nuevos sitios. Los ambientes desérticos, tanto fríos como cálidos, se caracterizan por tener agua disponible durante breves periodos, lo que tiende a favorecer a aquellas especies capaces de responder rápidamente a recursos efímeros (Ellis-Evans \& Walton 1990). Las características de las especies y del micrositio son claves para una colonización exitosa, determinando así que el éxito de colonización dependa no sólo del porcentaje de germinación, sino también de condiciones abióticas menos rigurosas que permitan a los organismos crecer y establecerse (Haussmann et al. 2010).

En general, nuestros resultados sugieren que $C$. quitensis es una especie plástica para todos los atributos medidos en el experimento de cambio climático simulado. De manera paralela, y no excluyente, nuestros resultados también sugieren que los individuos de $C$. quitensis provenientes de los diferentes sitios presentarían diferenciación ecotípica, respondiendo de manera consistente con las características ambientales de sus respectivos sitios de origen. Por ejemplo, el desempeño fisiológico ( $\mathrm{Fv} / \mathrm{Fm})$ fue mayor en la condición de cambio climático simulado en comparación a la condición normal, pero siendo sólo evidente en aquellos individuos provenientes del sitio más árido. Esto último sugeriría que aquellos individuos presentes en el sitio más árido (Arctowski) están expuestos a una condición de estrés que impide alcanzar un desempeño físiológico mayor, y que bajo un aumento en la disponibilidad hídrica permitiría un mejor desempeño de la maquinaría fisiológica. Por otro lado, individuos de ambos sitios presentaron un aumento tanto del ancho como del largo foliar, siendo esta variación más evidente en aquellos individuos provenientes del sitio más árido (Arctowski). Se ha demostrado, que el formar hojas de menor tamaño es una característica de especies que habitan en zonas con baja disponibilidad hídrica, debido a que disminuyen la pérdida de agua por evaporación (Nobel 2005). Por lo anterior, se sugiere que los individuos de ambos sitios expresan esta característica como una estrategia efectiva para disminuir los efectos negativos del estrés hídrico característico de la Antártica, siendo más evidente en aquellos individuos provenientes del sitio más árido. Consistente con nuestros resultados, Gianoli et al. (2004) trabajando con C. quitensis provenientes de la Antártica y Los Andes de Chile central, demostraron que esta especie presenta evidencia de diferenciación ecotípica en diversos atributos morfológicos y fisiológicos medidos.

Tras ser expuestos a una mayor disponibilidad hídrica, los individuos de C. quitensis provenientes de ambos sitios mostraron un aumento en el porcentaje de floración. No obstante, aquellos provenientes del sitio más árido (Arctowski) mostraron un aumento de mayor magnitud en 
comparación a sus con-específicos provenientes del sitio más húmedo (Hannah). Algunos estudios han mostrado que plasticidad en el esfuerzo reproductivo es una respuesta común en especies sometidas a estrés abiótico (Fox 1990, Passioura 2006). Estudios realizados con C. quitensis han demostrado que la floración es irregular en diversos sitios de la Antártica Marítima (Edwards 1972), lo cual se explica por la alta heterogeneidad abiótica de los sitios. La mayoría de las plantas de hábitat de tundras suelen reproducirse vegetativamente (Bliss 1971), pero se ha descrito que bajo ciertas condiciones ambientales menos rigurosas la reproducción vegetativa y la reproducción sexual pueden ser igualmente exitosas (Callaghan \& Emanuelsson 1985). Se ha demostrado que en dos especies de gramíneas que crecen en las islas sub-antárticas, el establecimiento a partir de semillas es la forma principal de reproducción (Grulke \& Bliss 1988) y que las poblaciones del pasto Phleum alpinum en la Isla Disko, en el Ártico, dependen principalmente de la reproducción sexual incluso si la producción de semillas es baja (Callaghan 1974). No obstante, la producción de semillas y el establecimiento de plántulas de $C$. quitensis es muy irregular, siendo el reclutamiento principalmente a través de reproducción vegetativa (Convey 1996). Si bien la producción de flores en C. quitensis ocurre todos los años, sólo ocasionalmente se han registrado semillas maduras y viables (Holtom \& Greene 1967, Edwards 1974).

En plantas de zonas árticas y antárticas el reclutamiento está limitado por bajas temperaturas que restringen la germinación, y por la mortalidad temprana de las plántulas a causa, principalmente, del estrés hídrico (Billings 1987, McGraw 1997). Es de esperar que la combinación de estaciones de crecimiento más largas con temperaturas más elevadas, y buena disponibilidad hídrica, afecten de manera positiva tanto a la maduración de las semillas como su germinación. Como se muestra en este estudio, la mayor disponibilidad hídrica aumenta de manera significativa el desempeño fisiológico y el esfuerzo reproductivo de C. quitensis, lo cual sumado a una mayor proporción de germinación de semillas y una menor mortalidad (ver Billings 1987), podría determinar un mayor éxito de colonización de nuevas áreas bajo un escenario futuro de cambio climático. Adicionalmente, se ha sugerido que el tamaño de los individuos es un buen bio-indicador de los efectos positivos del cambio climático (Fowbert \& Smith 1994). Por lo anterior, el hallazgo de individuos de $C$. quitensis de mayor tamaño en el sitio más húmedo (Hannah) podría indicar que las condiciones abióticas han mejorado por los efectos del cambio climático. Existe evidencia sustancial para sugerir que la dinámica de las comunidades vegetales antárticas están cambiando en respuesta al cambio climático global (Robinson et al. 2003, Wasley et al. 2006). Por ejemplo en las islas Signy, en las Orcadas del Sur, se ha demostrado un aumento en la temperatura y una reducción en la cobertura de hielo, lo que ha proporcionado a la vegetación nuevas áreas disponibles para la colonización (Smith, 1994). Esto último ha repercutido en cambios positivos en la abundancia y cobertura de C. quitensis en los últimos años (Fowbert \& Lewis Smith 1994).

Es muy probable que los impactos del aumento en la disponibilidad de agua sean especie-específicos y variables a lo largo de los diferentes ecosistemas antárticos (Robinson et al. 2003, Convey et al. 2008). Mientras algunos estudios han comparado las respuestas fisiológicas de diferentes especies antárticas en sitios con variación natural en la disponibilidad hídrica (ver, Lee et al. 2009), son escasos los estudios dirigidos a evaluar las respuestas funcionales ante las variaciones climáticas futuras. Si bien estamos lejos de comprender a cabalidad los efectos del cambio climático global sobre los procesos de colonización, este estudio proporciona los primeros indicios para comprender las respuestas de $C$. quitensis en el ecosistema antártico frente a futuros incrementos de la disponibilidad hídrica como consecuencia del cambio climático global.

\section{AGRADECIMIENTOS}

Agradecemos a Natalia Ricote-Martínez por su ayuda en las mediciones de terreno y al apoyo del personal técnico del Bio-Tecza por su colaboración durante el desarrollo del experimento de plasticidad. Agradecemos el financiamiento y apoyo logístico del Instituto Antártico Chileno (INACH proyecto T-14-08).

\section{BIBLIOGRAFÍA}

Beyer, L., M. Bölter \& R.D. Seppelt. 2000. Nutrient and thermal regime, microbial biomass and vegetation of Antarctic soils in the Windmill Islands region of east Antarctica (Wilkes Land). Arctic, Antarctic and Alpine Research 32: 30-39.

Billings, W.D. 1987. Constraints to plant growth, reproduction, and establishment in arctic environments. Arctic and Alpine Research 16: 357-365.

BLIss, L.C. 1971. Arctic and alpine plant life cycle. Annual Review of Ecology and Systematic 2: 405-438.

Callaghan, T.V. 1974. Intraspecific variation in Phleum alpinum with specific reference to polar populations. Arctic and Alpine Research 6: 361-401.

Callaghan, T.V. \& U. Emanuelsson. 1985. Population structure and processes of tundra plants an vegetation In: J. White (ed.), The Population Structure of Vegetation: 399-439. Dordrecht, Junk.

Convey, P. 1996. The influence of environmental characteristics on the life history attributes of Antarctic terrestrial biota. Biological Reviews 71: 191-225.

Convey, P. 2003. Maritime Antarctic climate change: signals from terrestrial biology. Antarctic Research Series 79: 145-158.

Convey, P., J.A.E. Gibson, C.D. Hillenbrand, D.A. Hodgson, P.J.A. Pugh, J.L. Smellie \& M.I. Stevens. 2008. Antarctic 
Plasticidad fenotípica en Colobanthus quitensis: Molina-Montenegro, M. ET AL.

terrestrial life - challenging the history of the frozen continent? Biological Review 83: 103-117.

Cullather, R.I., D.H. Bromwich \& M.L. van Woert. 1998. Spatial and temporal variability of Antarctic precipitation from atmospheric methods. Journal of Climate 11:334-367.

Edwards, J.A. 1972. Studies in Colobanthus quitensis (Kunth) Bartl. and Deschampsia antarctica Desv.: V. Distribution, ecology and vegetative performance on Signy Island. British Antarctic Survey Bulletin 28: 11-28.

Edwards, J.A. 1974. Studies on Colobanthus quitensis (Kunth) Bartl. and Deschampsia antarctica Desv. VI - Reproductive performance on Signy Island. British Antarctic Survey Bulletin 39: 67-86.

Ellis-Evans, J.C. \& D. Walton. 1990. The process of colonization in Antarctic terrestrial and freshwater ecosystems. Polar Biology 3: 151-163.

Ellstrand, N.C. \& K.A. Schierenbeck. 2000. Hybridization as a stimulus for the evolution of invasiveness in plants. Proceedings of the National Academy of Sciences 97: 7043-7050.

Flexas, J., M. Ribas-Carbó, J. Bota, J. Galmés, M. Henckle, S. Martínez-Cañellas, H. Medrano. 2006. Decreased Rubisco activity during water stress is not induced by decreased relative water content but related to conditions of low stomatal conductance and chloroplast $\mathrm{CO}_{2}$ concentration. New Phytologist 172: 73-82.

Fowbert, J.A. \& R.I.L. Smith. 1994. Rapid population increases in native vascular plants in the Argentine Island, Antarctic Peninsula. Arctic, Antarctic and Alpine Research 26: 290296.

Fox, G.A. 1990. Drought and the evolution of flowering time in desert annuals. American Journal of Botany 77: 15081518.

Gianoli, E., P. Hinostroza, A. ZúÑiga-Feest, M. Reyes-Díaz, L.A. Cavieres, L.A. Bravo \& L.J. Corcuera. 2004. Ecotypic differentiation in morphology and cold resistance in populations of Colobanthus quitensis (Cariophyllaceae) from the Andes of central Chile and the maritime Antarctic. Arctic, Antarctic, and Alpine Research 36: 484-489.

Grace, J. 1997. Plant water relations. In: M.J. Crawley (ed.), Plant ecology: 72-105. Oxford (UK), Blackwell.

Grulke, N.E. \& L.C. Bliss. 1988. Comparative life-history characteristics of two high Arctic grasses, Northwest Territories. Ecology 69: 484-496.

Haussmann, N.S., M.A. McGeoch \& J.C. Boelhouwers. 2010. Contrasting nurse plants and nurse rocks: The spatial distribution of seedlings of two sub-Antarctic species. Acta Oecologica 36: 299-305.

Holtom, A. \& S.W. Greene. 1967. The growth and reproduction of Antarctic flowering plants. Philosophical Transactions of the Royal Society, B 252: 323-337.

IPCC. 2007. Intergovernmental panel on climate change. http:// www.ipcc.ch

Kappen, L. 1999. Pflanzen und Mikroorganismen in der Polar regionen. 30 Jahre deutsche Beitrage zur Polarforschung. Naturwissenchaften Rundschau 52: 174-183.

KeJnA, M. 2008. Topoclimatic conditions in the vicinity of the Arctowski Station (King George Island, Antarctica) during the summer season of 2006/2007. Polish Polar Research 29: $95-116$.
Kennedy, A. D. 1993. Water as a limiting factor in the Antarctic terrestrial environment: A biogeographical synthesis. Arctic and Alpine Research 25: 308-315.

KomÁrková, V., S. Poncet \& J. Poncet. 1985. Two native Antarctic vascular plants, Deschampsia antarctica and Colobanthus quitensis: a new southernmost locality and other localities in the Antarctic Peninsula area. Arctic and Alpine Research 17: 401-416.

Lee, Y., H.S.Lim \& H. Yoon. 2009. Carbon and nitrogen isotope composition of vegetation on King George Island, maritime Antarctic. Polar Biology 32: 1607-1615.

Leishman, M.R. \& C. Wild. 2001. Vegetation abundance and diversity in relation to soil nutrients and soil water content in Vestfold Hills, East Antarctica. Antarctic Science 13: 126-134.

Maron, J.L., M. Vilà, R. Bommarco, S. Elmendorf \& P. Beardsley. 2004. Rapid evolution of an invasive plant. Ecological Monographs 74: 261-280.

Maxwell, A. \& G.N. Johnson. 2000. Chlorophyll fluorescence - a practical guide. Journal of Experimental Botany 345: 659668.

McGraw, J.B. 1997. Size and characteristics of a natural seed bank in Antarctica. Arctic and alpine Research 29: 213-216.

Molina-Montenegro, M.A. \& L.A. Cavieres. 2010. Variación altitudinal de los atributos morfo-fisiológicos en dos especies de plantas alto-andinas y sus implicancias contra la fotoinhibición. Gayana Botánica 67: 1-11.

Molina-Montenegro, M.A., A. Zurita-Silva \& R. Oses. 2011 a. Effect of water availability on physiological performance and lettuce crop yield (Lactuca sativa). Ciencia e Investigación Agraria 38: 65-74.

Molina-Montenegro, M.A., C.L. Quiróz, C. Torres-Díaz \& C. Atala. 2011 b. Functional differences in response to drought in the alpine invasive Taraxacum officinale from native and introduced habitat ranges. Plant Ecology \& Diversity 4: 37-44.

Nobel, P.S. 2005. Physicochemical and environmental plant physiology. Academic Press, New York.

Olech, M. \& K.J. ChwedorzewsKa. 2011. The first appearance and establishment of an alien vascular plant in natural habitats on the forefield of a retreating glacier in Antarctica. Antarctic Science 23: 153-154.

Passioura, J.B. 2006, Increasing crop productivity when water is scarce-from breeding to field management. Agricultural Water Management 80: 176-196.

Pedrol, N., P. Ramos, M.J. Reigosa. 2000. Phenotypic plasticity and acclimation to water deficits in velvet-grass: A longterm greenhouse experiment. Changes in leaf morphology, photosynthesis and stress-induced metabolites. Journal of Plant Physiology 157: 383-393.

Pigliucci, M. 2001. Phenotypic Plasticity: Beyond Nature and Nurture. John Hopkins University Press, Baltimore.

Rajakaruna, N., G.E. Bradfield, B.A. Bohm \& J. Whitton. 2003. Adaptive differentiation in response to water stress by edaphic races of Lasthenia californica (Asteraceae). International Journal of Plant Sciences 164: 371-376.

Robinson, S.A., J. Wasley \& A.K. Tobin. 2003. Living on the edge - plants and global change in continental and maritime Antarctica. Global Change Biology 9: 1681-1717.

Sexton, J.P., J.K. McKay \& A. Sala. 2002. Plasticity and genetic 
diversity may allow saltcedar to invade cold climates in North America. Ecological Applications 12: 1652-1660.

SмITH, R.I.L. 1994. Vascular plants as bioindicators of regional warming in Antarctica. Oecologia 99: 322-328.

Singh, S.M., N. Pereira \& Ravindra R. 2009. Adaptive mechanisms for stress tolerance in Antarctic plants. Current Science 99: 334-340.

Smith, R.I.L. 2003. The enigma of Colobanthus quitensis and Deschampsia antarctica in Antarctica. In: A.H.L. Huiskes, W.W.C Gieskes, J. Rozema, R.M.L. Schorno, S.M. van der Vies \& W.J. Wolff (eds.), Antarctic Biology in a Global Context: 234-239. Backhuys Publishers, Leiden, The Netherlands.
Turner, J., S.R. Colwell, G.J. Marshall, T.A. Lachlan-Cope, A.M. Carleton, P.D. Jones, V.L. Phil, A. Reid \& S. Iagovkina. 2005. Antarctic climate change during the last 50 years. International Journal of Climatology 25: 279-294.

Wasley, J., S.A. Robinson, C.E. Lovelock \& M. Popp. 2006. Climate change manipulations show Antarctic flora is more strongly affected by elevated nutrients than water. Global Change Biology 12: 1800-1812.

Ye, H. \& J.R. Mather. 1997. Polar snow cover changes and global warming. International Journal of Climatology 17: 155-162.

ZAR, J.H. 1999. Biostatistical analysis 4th edn. New Jersey: Prentice-Hall, Upper Saddle River.

Recibido: 13.05 .11

Aceptado: 09.12.11 